\section{NP45 (continued)}

culture of healthful eating and physical activity to prevent childhood obesity in young children.

Description: A collaborative, multi-state, multi-disciplinary team (IN, KS, MI, OH, SD, ND, WI) identified fourteen volunteer communities with existing community coalitions (seven were intervention and seven were comparative). Each completed Child Ecological Model assessments of their environments (Community- at-large, Early Childhood, Neighborhood, Active Where?) and coalitions annually selected nutrition and physical activity objectives from a team developed "menu" of evidence based/ evidence-informed approaches to prevent childhood obesity. Community Coaches, working in only the intervention communities, supported and promoted community capacity building through work directly with the coalitions.

Evaluation: With qualitative and quantitative data, the research examines the effectiveness of a community coaching model in improving the ability of the communities and coalitions to address or improve the needs identified in the community assessments. Overall, comparisons will be made between assessment findings from intervention and comparative communities with determination of the effectiveness of coached and non-coached communities in achieving change or enhancement of their environments and/or policies to prevent childhood obesity in 4-year olds. Final data analysis has been initiated in this last year of the project with collected community assessment data as well as annual Community Coalition SelfAssessments. Additionally, a Mind Mapping (Ripple Effect) exercise was conducted with each coalition and individual interviews of coaches and coalition members will also be analyzed.

Conclusions and Implications: Community capacity building with a socio-ecological basis and coaching will result in greatest changes in nutrition and physical activity behaviors among those caring for young children.

Funding: USDA Grant 2011-68001-30100

\section{NP46 Technology and Design Innovation to Support 21st Century School Nutrition}

Lorrene Ritchie,PhD, RD, LRitchie@ucanr.edu, Nutrition Policy Institute, University of California, 1111 Franklin, \#10123, Oakland, CA 94607

Objective: To assess the impact of innovative, studentcentered strategies based on behavioral economics to increase school lunch participation and intake of fruits and vegetables by middle and high school students in the San Francisco Unified School District in California.

Description: Using a cluster-randomized controlled trial design, we will assess the 2-year impact of implementing a 3-pronged intervention involving a SmartMeal technology platform, distributed points of sale, and staff promotion of school meals on school lunch participation and intake of fruits and vegetables by 7 th-10th grade students from 12 middle and high schools compared to 12 control schools.
Evaluation: The primary outcome is the difference in change over two years in average daily participation in school lunch between intervention and control schools. We will use a linear regression model in order to adjust for school enrollment, free and reduced price school meal enrollment, and race/ethnic composition.

Conclusions and Implications: Several unanticipated challenges have delayed implementation of the intervention and its subsequent evaluation. These challenges are similar to those faced by many large, urban school districts seeking to implement a project of this scope, and thus provide important insight for districts seeking to implement similar interventions.

Funding: USDA Grant \#2015-68001-23236

\section{NP47 The Pennsylvania State University Childhood Obesity Prevention Graduate Training Transdisciplinary Program}

Barbara Rolls,PhD, bjr4@psu.edu, Pennsylvania State University, 226 Henderson Building, University Park, PA 16802; J. Savage, PhD; K. Keller, PhD

Objective: This training program prepares pre-doctoral fellows for careers in childhood obesity prevention through transdisciplinary coursework, research, and internships.

Description: This program includes four programmatic areas, including nutritional sciences; child development and family studies; prevention and intervention, and research methodology and statistics.

Evaluation: The evaluation is accomplished through yearly external advisory meetings; program evaluation from trainees; ongoing publications and professional accomplishments of trainees, and biweekly meetings of key personnel.

Conclusions and Implications: Thirteen fellows have produced 92 presentations and 32 peer reviewed publications in the first 5 years of the program.

Funding: USDA Grant \#2011-67001-30117

\section{NP48 Niños Sanos, Familia Sana - Impact on Child Physical Activity}

SaraSchaefer,PhD, seschaefer@ucdavis.edu, University of California Davis, 2141 RMI North Building, Davis, CA 95616; B. Sadeghi, MD, PhD; L. Whent, PhD; A. de la Torre, PhD

Objective: To fill a gap in knowledge related to effective interventions in Mexican-heritage populations, this project had four aims: slow down the rate of weight gain among intervention children compared to controls; identify mediating factors related to economic and nutrition education intervention components; develop a culturally-adapted nutrition and physical activity curriculum for families; and increase cultural competency of university students. The primary objective of this paper is to 